(C) 2021, The Authors. Published by Elsevier Inc. and Fass Inc. on behalf of the American Dairy Science Association ${ }^{\circledR}$. This is an open access article under the CC BY-NC-ND license (http://creativecommons.org/licenses/by-nc-nd/4.0/).

\title{
Whey protein addition and its increased light absorption and tinctorial strength of model solutions colored with anthocyanins
}

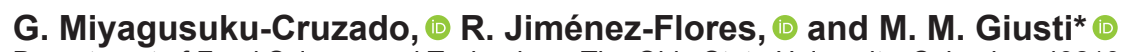 \\ Department of Food Science and Technology, The Ohio State University, Columbus 43210-1007
}

\begin{abstract}
Anthocyanins $(\mathrm{ACN})$ are pigments with vivid colors, but their application as food colorants is restricted by their limited stability and color expression. Anthocyanins exhibit higher stability in dairy systems than in buffers at similar $\mathrm{pH}$, suggesting that pigments may be able to interact with dairy components such as proteins, resulting in improved performance as colorants. Our objective was to determine the type of interaction between whey proteins (WP) and ACN leading to color enhancements and to determine the role of the ACN chemical structure in this interaction. Model solutions colored with semipurified pigments from sources with different ACN profiles (Berberis boliviana, grape skin, purple corn, black carrot, and red cabbage) were mixed with different concentrations of whey protein isolate (WPI) in $\mathrm{pH} 3$ buffer. Absorption spectra of these solutions were acquired using an absorbance microplate reader, and color parameters were calculated from spectral data. Isolated ACN 3-glucosides were used to determine the role of the aglycone structure in the WP-ACN interaction using visible and fluorescence spectroscopy. In silico modeling was used to visualize potential differences in the interaction between $\beta$-lactoglobulin and ACN. Addition of WPI resulted in hyperchromic shifts at the wavelength of maximum absorption in the visible range $\left(\lambda_{\text {vis-max }}\right)$ of up to $19 \%$, and a significant increase in tinctorial strength for all $\mathrm{ACN}$ sources $(\Delta \mathrm{E}>5)$. Moreover, $\mathrm{ACN}$ acylation did not seem to play a significant role in the WP-ACN interaction. When using isolated ACN, WPI addition resulted in hyperchromic shifts at the $\lambda_{\text {vis-max }}$ only for methoxylated ACN such as petunidin-3-glucoside (up to 24\%), and malvidin-3-glucoside (up to 97\%). The bimolecular quenching constant values $\left(K_{q}>10^{10} M^{-1} \mathrm{~s}^{-1}\right)$ strongly suggested that the predominant type of quenching interaction was static. Analysis of enthalpy, entropy, and Gibbs free energy showed that this binding was
\end{abstract}

Received September 23, 2020.

Accepted December 23, 2020.

*Corresponding author: giusti.6@osu.edu spontaneous; depending on the chemical structure of the ACN, the predominant binding forces could be hydrophobic interactions or hydrogen bonding. Modeling suggested that methoxylations in the B ring of the aglycon structure promoted interactions with electron acceptor amino acids. Overall, WP could be used to enhance the tinctorial strength of select ACN depending on their structural characteristics. Therefore, ACN source selection may play a key role for specific applications in dairy products.

Key words: anthocyanin, whey protein, complexation

\section{INTRODUCTION}

Whey proteins (WP) and phenolic compounds have been shown to interact, resulting in modifications in the functional properties of both proteins and phenolics (Ozdal et al., 2013). This interaction can produce changes in WP solubility, thermal stability, digestibility (Rawel et al., 2001; Cao and Xiong, 2017), antioxidant activity, and foaming and emulsifying properties (Ali et al., 2013; Rodríguez et al., 2015; Jia et al., 2016). Similarly, complexation with WP can result in changes in the functional characteristics of phenolic compounds, such as antioxidant activity, in vivo bioavailability, stability, and solubility (Rohn et al., 2004; Liang et al., 2008; Serafini et al., 2009; Chung et al., 2015), and the chemical structure of the phenolic compound interacting with WP (Ozdal et al., 2013). Although literature shows extensive research on the changes in functional properties of phenolic compounds in response to their complexation with WP, the information available on the influence of WP on the color expression of phenolic colorants is scarce.

The study of the color in food is important because color plays a key role in the consumer's identification, perception of quality, and subsequent acceptance or rejection of a product (Crisosto et al., 2003). Current trends show that the food industry is moving away from synthetic colorants due to potential behavioral concerns in children and an increased consumer awareness (Bateman et al., 2004; McCann et al., 2007). In this context, colorants from natural sources are becoming 
more prevalent because of increased acceptance by the market (Burrows, 2009; Sigurdson et al., 2017). Among these colorants, anthocyanins (ACN) are emerging as potential suitable replacements for synthetic dyes for food applications, due to their ability to impart vivid colors that can range from red to blue (Giusti and Wrolstad, 2003; Obón et al., 2009) and potential health benefits associated with their consumption (He and Giusti, 2010). Anthocyanins are water-soluble flavonoids with a $\mathrm{C} 6-\mathrm{C} 3-\mathrm{C} 6$ structure that have one or more glycosylations usually linked through $O$-glycosidic bonds (Wrolstad, 2004; Farr et al., 2018). Moreover, ACN can have organic acids attached to these glycosylations via ester bonds (Giusti and Wrolstad, 2003). Although the application of ACN as food colorants seems promising, challenges arise from their limited stability and color expression after processing and during storage, especially when used in matrices with $\mathrm{pH}$ close to neutral. An additional possible disadvantage is that ACN extracts from vegetable sources can impart undesirable odor or flavor characteristics (Giusti and Wrolstad, 2003). As $\mathrm{pH}$ increases from 1 to 4.5 , the structure of the ACN changes from the flavylium cation form into a colorless carbinol form as a result of a nucleophilic attack by water (Chen and Hrazdina, 1982; Santos et al., 1993). In theory, these color expression characteristics should severely hinder the application of ACN-rich extracts as food colorants in dairy matrices. However, ACN from purple corn (PC) cob has exhibited a higher resistance to thermal degradation in milk compared with a buffer at the same pH (Jing and Giusti, 2005). Similarly, ACN from Berberis boliviana (BB) were successfully used as a colorant in yogurt, with color characteristics and stability similar to the more common industrially used betalains (Wallace and Giusti, 2008). Moreover, studies have shown that addition of WP results in improvements of the shelf life of model juices colored with ACN while increasing their resistance to ascorbic acid bleaching (Chung et al., 2015; He et al., 2016a; Stănciuc et al., 2017).

The objective of the present study was to determine the type of interaction between $\mathrm{WP}$ and ACN that results in color enhancements using visible and fluorescence spectroscopy. Moreover, the effects of the chemical structure of ACN on their interaction with WP was also evaluated.

\section{MATERIALS AND METHODS}

\section{Materials}

Freeze-dried BB berries were donated by the Universidad Nacional San Antonio Abad del Cusco (Cusco, Peru), PC corncobs (Zea mays L.) by Globenatural
Intl. SA, and grape skin (GS) extract was obtained from Polyphenolics Inc. Strawberries, red cabbages (RC), and black carrots (BC) were purchased from grocery stores in Columbus, Ohio. For individual pigment isolation, strawberry was selected as a source of pelargonidin-3-glucoside (pg-3-glu), PC was selected as a source of cyanidin-3-glucoside (cy-3-glu), and $\mathrm{BB}$ was selected for isolation of delphinidin-3-glucoside (dp-3-glu), petunidin-3-glucoside (pt-3-glu), and malvidin-3-glucoside ( $\mathbf{m v - 3 - g l u})$. Whey protein isolate (WPI) was purchased from Hilmar Ingredients. All chemicals and solvents were of American Chemical Society analytical or ultra-HPLC grade unless otherwise indicated.

\section{ACN Extraction and Purification}

Extractions of ACN from plant samples were conducted following the methodology published by RodriguezSaona and Wrolstad (2001). Briefly, plant samples were powdered using liquid nitrogen and treated with $0.01 \%$ $\mathrm{HCl}$ acidified in $70 \%$ aqueous acetone (vol/vol). The slurry was filtered through a Buchner funnel, and the cake was re-extracted with acidified aqueous acetone until a clear solution was observed. The pigment-rich aqueous acetone filtrates were partitioned with chloroform in a proportion of 1:2 acetone:chloroform ( $\mathrm{vol} /$ vol) and stored overnight at $4^{\circ} \mathrm{C}$. The aqueous portion was then collected, and residual acetone was removed using a rotary evaporator at $40^{\circ} \mathrm{C}$. The $\mathrm{ACN}$ crude extracts were then semipurified using the methodology described by Rodriguez-Saona and Wrolstad (2001). Briefly, crude ACN extracts were passed through a $\mathrm{C}_{18}$ column preactivated with 3 volumes of methanol and 3 volumes of $0.01 \% \mathrm{HCl}(\mathrm{vol} / \mathrm{vol})$ acidified water. The ACN were adsorbed to the column, after which sugars, acids, and other water-soluble compounds were eluted with 2 volumes of acidified water. Other polyphenolic compounds were washed away using 2 volumes of ethyl acetate. Adsorbed ACN were then eluted using $0.01 \%$ (vol/vol) $\mathrm{HCl}$ acidified methanol. Methanol was evaporated using a rotary evaporator at $40^{\circ} \mathrm{C}$, and pigments were reconstituted with acidified water.

\section{Alkaline Hydrolysis of Acylated ACN}

Saponification of ACN was conducted following the methodology described by Giusti and Wrolstad (1996). Briefly, semipurified ACN extracts were hydrolyzed by diluting them with $10 \%$ aqueous $\mathrm{KOH}$ for $8 \mathrm{~min}$ at room temperature in glass vials. Solutions were then neutralized using $2 \mathrm{~N} \mathrm{HCl}$. Hydrolysate was semipurified again using solid phase extraction to remove organic acids and salts produced during this step. 


\section{ACN Isolation}

Individual ACN were isolated from semipurified extracts using semipreparative HPLC, following the methodology of Farr et al. (2018). The system consisted of 2 LC-6AD pumps, a CMB-20A communication module, a SIL-20AHT autosampler, a CTO-20A column oven, and an SPD-M20A photodiode array detector (Shimadzu). The column used for chromatographic separation of ACN was a $5-\mu \mathrm{m}$ particle size Luna pentafluorophenyl $21.2 \times 250 \mathrm{~mm}$ (Phenomenex) column with a binary solvent system consisting of A: $4.5 \%$ formic acid acidified water (vol/vol) and B: $100 \%$ acetonitrile. After isolation, ACN were semipurified and concentrated using solid phase extraction with $\mathrm{C}_{18}$ cartridges, following the methodology described previously without the ethyl acetate wash step.

\section{Monomeric ACN Quantification}

Monomeric ACN content was calculated using the pH-differential method (Giusti and Wrolstad, 2001). A SpectraMax M2 plate reader (Molecular Devices) was used to measure the maximum absorbance within the visible range $\left(\boldsymbol{\lambda}_{\text {vis-max }}\right)$ and $700 \mathrm{~nm}$. Results were expressed as cyanidin-3-glucoside equivalents, using an extinction coefficient of $26,900 \mathrm{~L} \mathrm{~mol}^{-1} \mathrm{~cm}^{-1}$ and a molecular weight of $449.3 \mathrm{~g} / \mathrm{mol}$.

\section{HPLC with Photodiode Array Detection and Electrospray Interface Tandem MS Profiling of ACN and Purity Check of Isolated ACN}

Analysis and tentative identification of $\mathrm{ACN}$ were carried out on semipurified extracts and isolated ACN. Briefly, an aliquot of $5 \mu \mathrm{L}$ was injected into a Nexera-iLC-2040 3D ultra-HPLC system (Shimadzu). The system consisted of 4 pumps, a refrigerated autosampler, a column oven, and a photodiode array detector coupled to an LCMS-8040 triple quadrupole mass spectrometer with electrospray interface. Separation of ACN was achieved using reverse-phase chromatography under identical conditions. The column used was a Kinetex F5 column of $1.7 \mu \mathrm{m} \times 2.1 \mathrm{~mm} \times 100 \mathrm{~mm}$ (Phenomenex), and solvents were A: $4.5 \%$ formic acid acidified water (vol/vol) and B: 100\% acetonitrile. Separation of ACN was achieved at $50^{\circ} \mathrm{C}$ using the following gradient: $3 \%$ B for 0 to $1.75 \mathrm{~min}, 10 \%$ from 3.5 to $9 \mathrm{~min}, 10$ to $40 \%$ from 9 to $17 \mathrm{~min}$, and $40 \%$ from 17 to $19 \mathrm{~min}$. Spectral data were collected from 250 to $700 \mathrm{~nm}$ over the whole time of the run. Mass spectra and collision-induced dissociation data were acquired under positive mode with $1.5 \mathrm{~L} / \mathrm{min}$ nebulizing gas flow, $15 \mathrm{~L} / \mathrm{min}$ of drying gas, desolvation gas temperature of $230^{\circ} \mathrm{C}$, and a collision energy of $35 \mathrm{eV}$. Data were monitored using total ion scan from 100 to $1,000 \mathrm{~m} / z$, and precursor ion scan was conducted for $271,287,301,303,317$, and $331 \mathrm{~m} / \mathrm{z}$, representing the 6 common anthocyanidins found in food. Data analysis was performed using Lab Solutions Software version 1 (Shimadzu).

\section{Preparation of Model Solutions and Visible Spectroscopy Experiments}

Model solutions were prepared by diluting ACN extracts and a concentrated WPI stock $(20 \mathrm{mg} / \mathrm{mL})$ with citric acid $\mathrm{Na}_{2} \mathrm{HPO}_{4}$ buffer at $\mathrm{pH}$ 3. Control proteinfree solutions were diluted until an absorbance of $\sim 0.6$ was obtained. Model solutions containing proteins were formulated to reach a final WPI concentration of 0 , $0.01,0.05,0.1,0.5$, and $1 \mathrm{mg} / \mathrm{mL}$. The $\mathrm{pH}$ of the final solutions was adjusted to 3.0 using $1 M$ citric acid or 1 $M \mathrm{Na}_{2} \mathrm{HPO}_{4}$, when needed. Solutions stood for $15 \mathrm{~min}$ to allow for stabilization, after which absorption measurements were conducted. Briefly, aliquots of $250 \mu \mathrm{L}$ were placed on 96-well plates, and light absorption was measured between 380 and $700 \mathrm{~nm}$ using a SpectraMax M2 plate reader (Molecular Devices).

\section{Color Parameter Calculations}

Color parameters of model solutions colored with ACN were calculated and expressed in the CIE L* $\mathrm{c}^{*} \mathrm{~h}^{*}$ communication system using the ColorBySpectra software developed by Farr and Giusti (2017). Parameters were calculated using CIE conversion equations for D65 illuminant and $10^{\circ}$ observed angle. Color parameters were used to calculate total color change $(\Delta \mathrm{E})$ using the CIE L*a*b* Delta E2000 equation (Mokrzycki and Tatol, 2011).

\section{Fluorescence Quenching Experiments}

Spectrofluorometric experiments were used to assess the type of interaction between WP and ACN. Briefly, samples containing $1 \mathrm{mg} / \mathrm{mL}$ WPI (mainly composed of $\beta$-LG) and different concentrations of isolated ACN $(1,5,10,25,50,75$, and $100 \mu M$ cyanidin-3-glucoside equivalents) were prepared using citric acid $\mathrm{Na}_{2} \mathrm{HPO}_{4}$ buffer at $\mathrm{pH}$ 3. The fluorescence intensities of solutions with different WPI-ACN ratios were measured at 303.15, 308.15, and 313.15 K using a Spectramax M2 plate reader (Molecular Devices). The selected excitation wavelength was $280 \mathrm{~nm}$, and emission spectra were collected from 360 to $500 \mathrm{~nm}$ with a step of $5 \mathrm{~nm}$. The Stern-Volmer equation was used to describe the quenching phenomena and to determine whether the 
reduction in fluorescence was a result of dynamic or static quenching.

\section{Molecular Docking Simulations}

In silico molecular docking simulations were used as complementary tools to further understand how ACN interact with $\beta$-LG. The crystalline structure of $\beta-\mathrm{LG}$ (2GJ5) was obtained from the Protein Data Bank (https://www.rcsb.org). Anthocyanin structures for pg-3-glu (ID: 443648), cy-3-glu (ID: 441667), dp-3-glu (ID: 443650), pt-3-glu (ID: 443651), and mv-3-glu (ID: 443652) were obtained from the PubChem database (https://pubchem.ncbi.nlm.nih.gov/). Docking experiments were conducted using mCule docking software (https://mcule.com/apps/1-click-docking/), which uses a built-in AutoDock Vina engine to perform docking of small ligands against a predetermined target site in the crystalized structure of the protein. The docking position with the lowest docking score was selected for further analysis. Visualization of potential binding residues in the amino acid sequence of $\beta$-LG was generated using PyMOL Molecular Graphics System (Schrödinger LLC).

\section{Statistical Analysis}

Regression analyses and one-way ANOVA with Bonferroni's post hoc tests were performed using GraphPad Prism (GraphPad Software Inc.). All experiments were conducted in triplicate. A $P$-value lower than 0.05 was considered significant.

\section{RESULTS AND DISCUSSION}

\section{Spectral and Color Changes in Response to WPI Addition to Model Solutions Colored with ACN}

Addition of WPI to model solutions colored with ACN resulted in hyperchromic shifts at the $\lambda_{\text {vis-max }}$ of up to $17.1 \pm 2.0 \%$ for BB, $19.9 \pm 2.2 \%$ for GS, $15.8 \pm$ $0.9 \%$ for $\mathrm{PC}, 9.9 \pm 0.3 \%$ for $\mathrm{BC}$, and $7.2 \pm 1.7 \%$ for $\mathrm{RC}$ (Figure 1). These absorption increases were significant for all ACN sources $(P<0.01)$. Figure 1 also shows linear regression plots for the increase in the relative absorbance at the $\lambda_{\text {vis-max }}$ as a function of the protein concentration for the original and the saponified extract. These plots showed that the absorption increases at the $\lambda_{\text {vis-max }}$ were concentration dependent. Consequently, regression analyses were carried out, with results shown in Table 1. These results confirmed that the hyperchromic shifts in response to WPI addition followed a linear model with relatively high coefficients of determination $\left(\mathrm{R}^{2}>0.75\right)$ for all anthocyanin sources except for the extracts from RC, which showed lower values. No significant differences were found when comparing the slopes of BB, GS, and PC $(P>0.05)$, suggesting that the absorption increase rates in response to WPI addition were similar among these ACN sources. However, these rates differed from $\mathrm{BC}$ and $\mathrm{RC}(P>$ 0.05 between $\mathrm{BC}$ and RC, $P<0.05$ against BB, GS, and PC). Therefore, hyperchromic shifts in response to WPI addition were concentration dependent, with results suggesting that not all ACN reacted in the same manner. Several studies have reported hyperchromic and bathochromic shifts as a result of ACN copigmentation with phenolic compounds such as catechins (Kanha et al., 2019), hydroxycinnamic acids (WilskaJeszka and Korzuchowska, 1996; Eiro and Heinonen, 2002; Kanha et al., 2019), hydroxybenzoic acids (Eiro and Heinonen, 2002), flavones (Chatham et al., 2020), and flavonols (Wilska-Jeszka and Korzuchowska, 1996; Chatham et al., 2020). Unlike copigmentation between ACN and phenolics, WPI addition to model solutions only resulted in hyperchromic shifts with no bathochromic shifts observed. This behavior is consistent with that reported for mulberry anthocyanins (Khalifa et al., 2018), where addition of WP also resulted in only hyperchromic shifts. The increase in the absorption at the $\lambda_{\text {vis-max }}$ in response to WP addition can be explained by the formation of a WP-ACN complex. This complexation may favor the formation of flavylium cations, thus disfavoring the colorless carbinol form that is predominant at pH 3 (Santos et al., 1993). Thereby, absorption at the $\lambda_{\text {vis-max }}$ increases as a result of the formation of flavylium cations due to the complexation of ACN with WP. Moreover, this phenomenon would also explain the absence of bathochromic or hypsochromic shifts in the spectra.

To study the effects of ACN acylation on the interaction between WP and ACN, similar experiments were carried out with saponified extracts. Results presented in Table 2 showed that the saponification process was successful, as no peaks corresponding to acylated ACN were found in the HPLC with photodiode array detection chromatogram after alkaline hydrolysis. Addition of WPI to model solutions colored with saponified ACN extracts also resulted in spectral changes comparable to those observed for the original extracts (Figure 1). These hyperchromic shifts were of up to $17.7 \pm 2.8 \%$ for saponified GS, $17.8 \pm 0.8 \%$ for saponified PC, 8.7 $\pm 0.6 \%$ for saponified $\mathrm{BC}$, and $5.0 \pm 1.2 \%$ for saponified RC. No significant differences were found when comparing the absorption increase rate of the original extract against its saponified counterpart $(P>0.05)$. This strongly suggests that ACN acylation did not significantly impact absorption changes after complexation with WP. Therefore, we hypothesized that the 
key structural traits of the ACN molecule that promote spectral changes in response to complexation with WP may reside in the aglycon moiety. This hypothesis is supported by data displayed in Table 2, showing that pigment sources rich in methoxylated ACN had larger hyperchromic shifts in response to WPI addition.

To better understand how these hyperchromic shifts resulted in color changes, color parameters were calculated from spectral data (Table 3). Addition of WPI resulted in noticeable color changes for all model solutions, regardless of the ACN source used. These changes were noticeable $(\Delta \mathrm{E}>5)$ at WPI concentrations as small as $0.05 \mathrm{mg} / \mathrm{mL}$ for $\mathrm{BB}, 0.5 \mathrm{mg} / \mathrm{mL}$ for GS, $0.5 \mathrm{mg} / \mathrm{mL}$ for $\mathrm{PC}$, and $1.0 \mathrm{mg} / \mathrm{mL}$ for $\mathrm{BC}$ and RC. All model solutions showed a significant decrease $(P<0.01)$ in lightness $\left(\mathrm{L}^{*}\right)$ and chroma $\left(\mathrm{c}^{*}\right)$ and a significant increase $(P<0.01)$ in their hue angle $\left(\mathrm{h}^{*}\right)$. This means that the solutions became darker, less bright, and less purple but with redder hues. Therefore, WPI addition resulted in model solutions with sig-
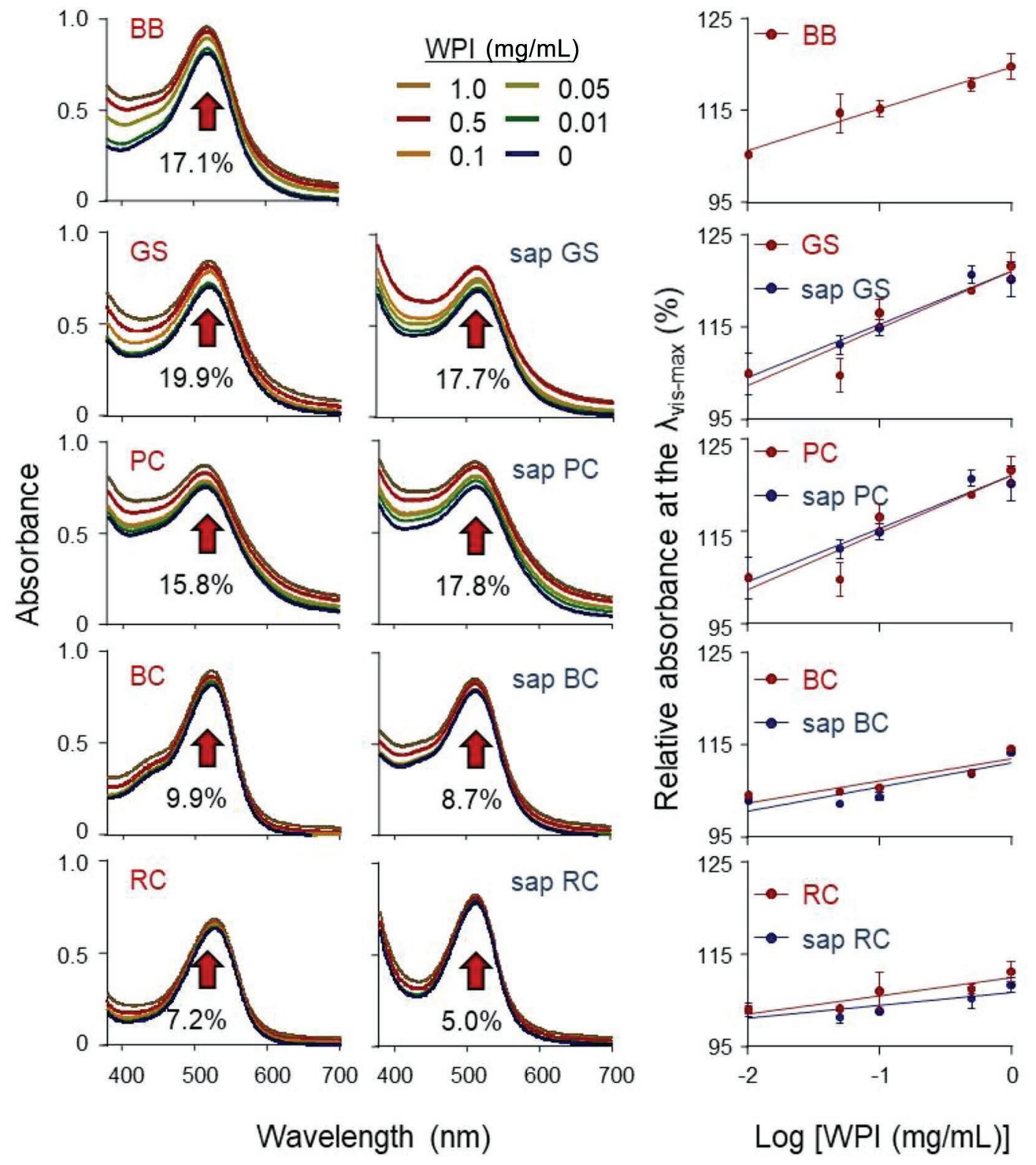

Figure 1. Spectral changes in response to whey protein isolate (WPI) addition to model solutions colored with semipurified anthocyanin (ACN)-rich extracts (left-hand column). Effect of saponification on spectral changes in response to WPI addition (middle column). Changes in relative absorbance at the visible range $\left(\lambda_{\text {vis-max }}\right)$ expressed as a percentage in response to WPI addition (right-hand column). Data show means of $\mathrm{n}=3$; bars represent $1 \mathrm{SD} . \mathrm{BB}=$ Berberis boliviana $\mathrm{ACN}, \mathrm{GS}=$ grape skin $\mathrm{ACN}, \mathrm{PC}=$ purple $\operatorname{corn} \mathrm{ACN}, \mathrm{BC}=$ black carrot $\mathrm{ACN}, \mathrm{RC}=$ red cabbage $\mathrm{ACN}$, sap = saponified. 
Table 1. Linear regression results obtained from plotting absorbance at the wavelength of maximum absorption in the visible range $\left(\lambda_{\text {vis-max }}\right.$, $\mathrm{Y}$ ), as a function of $\log _{10}$ of the concentration of whey protein isolate in $\mathrm{mg} / \mathrm{mL}$ (X, absorbance expressed as a percentage of the absorbance of a protein-free control)

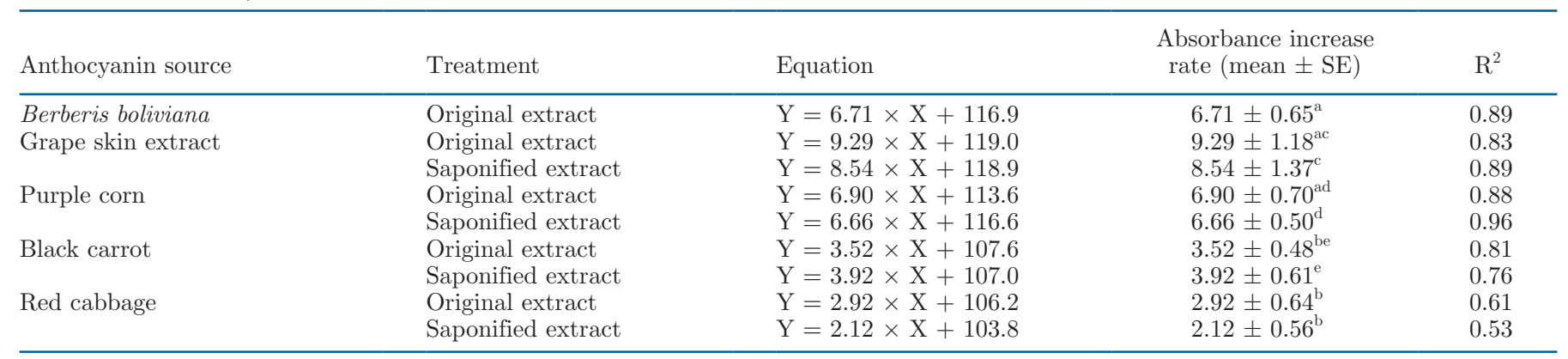

${ }^{\mathrm{a} e}$ Different letters indicate significant differences at the level of $P<0.05$.

nificantly higher light absorption and noticeably darker colors, thus increasing the tinctorial strength of model solutions. This agrees with previous results that also showed that addition of WP to mulberry juice resulted in deeper colors (Khalifa et al., 2018), suggesting that WP can act as a copigment.

\section{Study of the Role of the ACN Aglycon Chemical Structure in its Interaction with WP Using Visible Spectroscopy}

To determine whether the aglycone structural characteristics affected the WP-ACN interaction, similar experiments were conducted with isolated ACN and higher concentrations of WPI. Chromatographic separation and isolation of $\mathrm{ACN}-3$-glucosides from strawberries, $\mathrm{PC}$, and $\mathrm{BB}$ were successful, reaching high levels of purity ( $>90 \%$, data not shown). Consequently, it was assumed that any spectral change would occur mainly as a result of ACN interactions with WP. Results in Figure 2 showed that spectral changes in response to WPI addition were indeed dependent on the structure of the ACN aglycon. Model solutions colored with ACN with only hydrogenations or hydroxylations on positions $3^{\prime}$ and $5^{\prime}$ of the $\mathrm{B}$ ring showed a decrease in their absorptions at their $\lambda_{\text {vis-max }}$ in response to WPI addition. This decrease was of $8.2 \pm 5.1 \%$ for pg-3-glu, $5.0 \pm 5.4 \%$ for cy-3-glu, and $17.08 \pm 2.1 \%$ for dp-3glu. This phenomenon is called anti-copigmentation, and although it is rare, it is not unreported. Previous studies have reported similar hypochromic shifts resulting from the interaction between $\beta$-cyclodextrin and hydroxylated ACN, such as pg-3-glu (Yamada et al., 1980; Dangles et al., 1992b) and cy-3-glu (Yamada et al., 1980). It was then hypothesized that the interaction between $\beta$-cyclodextrin and ACN shifted the hydration equilibrium toward the formation of colorless forms (Dangles et al., 1992a), resulting in a decreased light absorption of the complex.
On the other side, Figure 2 illustrates that ACN with methoxylations on position $5^{\prime}$ or in both $3^{\prime}$ and $5^{\prime}$ showed increases in their absorption in response to WPI addition, of $24.1 \pm 1.4 \%$ for pt-3-glu and $97.1 \pm$ $40.2 \%$ for mv-3-glu. This is consistent with our experiments using semipurified extracts, where sources rich in methoxylated ACN, such as GS, BB, and PC (Table 2 ), showed faster absorption increase rates in response to WPI addition than sources without them, such as $\mathrm{BC}$ and RC (Figure 1 and Table 1). Similar results were found in published literature, showing that copigmented solutions colored with methoxylated ACN had larger absorption increases than solutions colored with nonmethoxylated ACN (Mazza and Brouillard, 1990; Eiro and Heinonen, 2002; Zhao et al., 2020).

\section{Study of the Role of the ACN Aglycon Chemical Structure in its Interaction with WP Using Fluorescence Spectroscopy}

Preliminary results showed that the shape of the fluorescence emission spectra of WPI was not affected by the presence of ACN, indicating that under our experimental conditions (e.g., $280 \mathrm{~nm}$ ), fluorescence emission was entirely dominated by the protein. Although it did not affect the spectral shape, the presence of ACN resulted in a decrease in the fluorescence emission intensity (data not shown). Intrinsic fluorescence in proteins is mainly attributed to tryptophan (Trp) and tyrosine (Tyr) amino acid residues; it has been reported that in $\beta-\mathrm{LG}$, the most abundant protein in whey, Trp19 is mainly responsible for its fluorescence (Albani et al., 2014). Literature has shown that the process of decreasing the fluorescence intensity of a sample, also called fluorescence quenching, is a useful analytic tool for the study of the interaction mechanics between phenolics and proteins (Hsiao et al., 2009; Zhang et al., 2014; Cao and Xiong, 2017; Wu et al., 2018). In this study, due to the high level of purity of 
Miyagusuku-Cruzado et al.: WHEY PROTEIN-ANTHOCYANIN COMPLEXATION

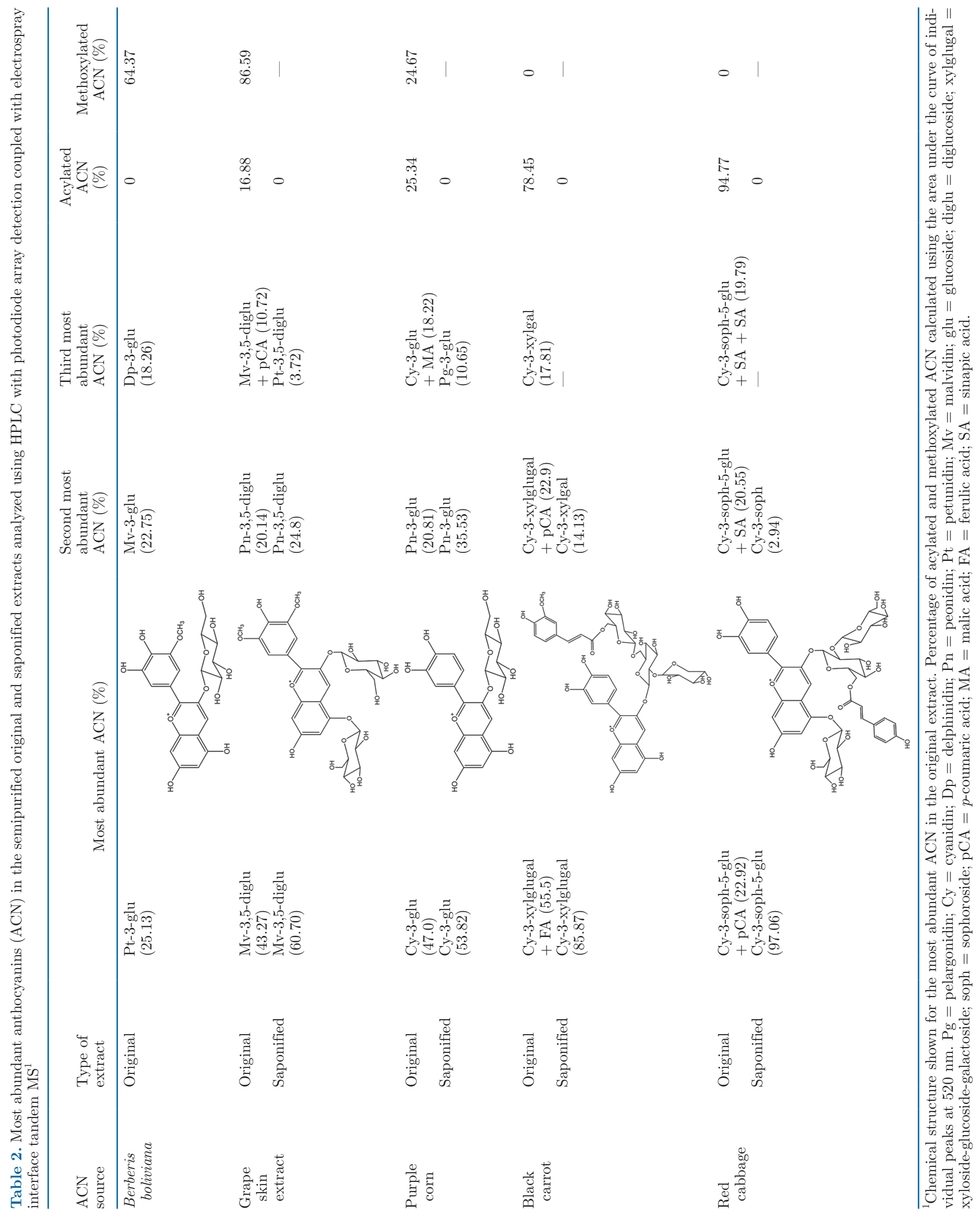




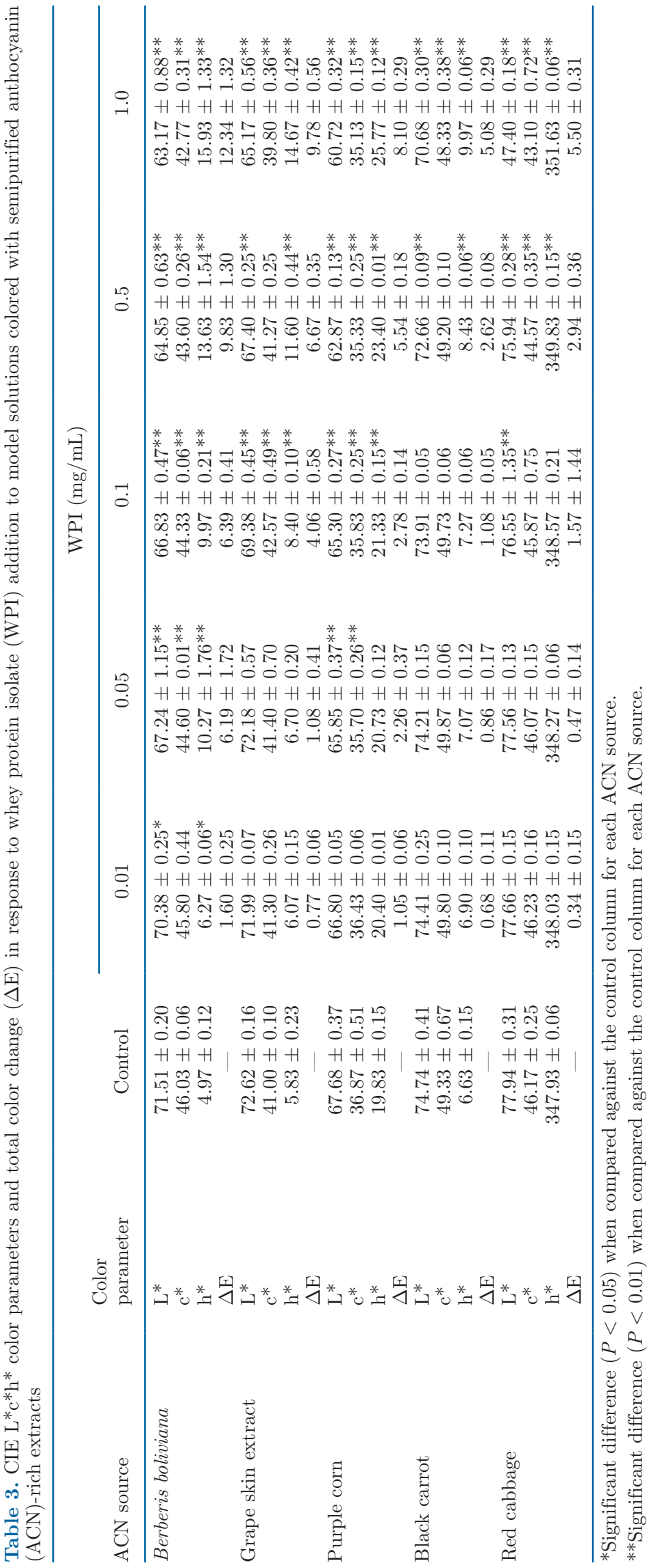



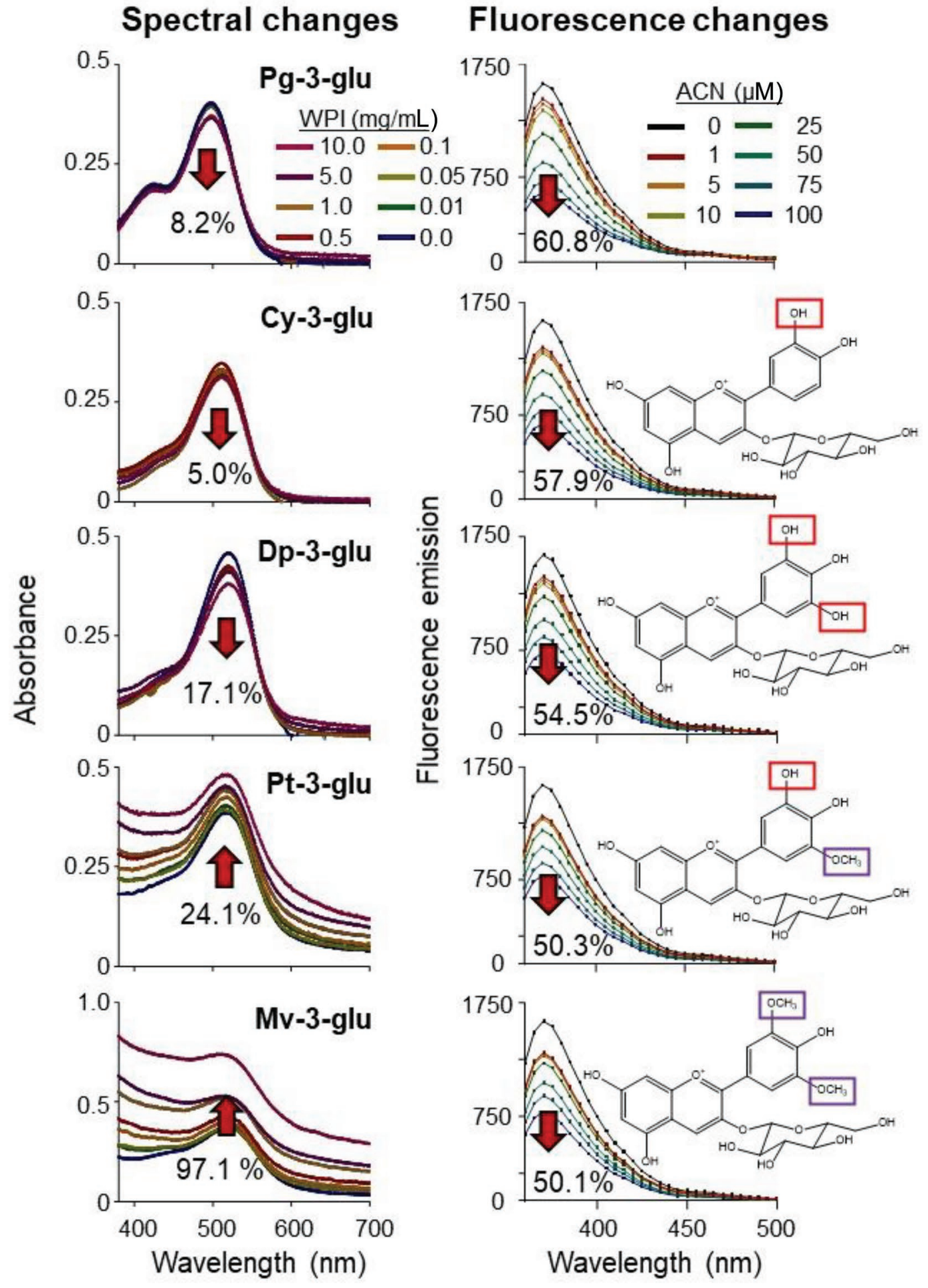

Figure 2. Spectral changes in response to whey protein isolate (WPI) addition to model solutions colored with isolated anthocyanins (ACN) using semi-preparative HPLC (left-hand column). Fluorescence quenching of a 1-mg/mL WPI solution in response to ACN addition (right-hand column). Data show means of $n=3$. Pg-3-glu = pelargonidin-3-glucoside, Cy-3-glu = cyanidin-3-glcoside, Dp-3-glu $=$ delphinidin-3-glucoside, Pt-3-glu = petunidin-3-glucoside, and Mv-3-glu = malvidin-3-glucoside. 
isolated $\mathrm{ACN}$, it was assumed that quenching of WP fluorescence would be a result of WP-ACN interactions rather than of interference by other components in the matrix. Results in Figure 2 showed that ACN addition resulted in a concentration-dependent quenching of WP fluorescence, suggesting some type of interaction between WP and ACN. The Stern-Volmer equation Eq. [1] was used as a mathematical model to describe the quenching of protein fluorescence in response to incremental concentrations of ACN. This model was also used to determine whether the type of quenching was due to collisional interactions (dynamic) or bindingrelated ones (static):

$$
\frac{\mathrm{F}_{0}}{\mathrm{~F}}=1+K_{S V}[\mathrm{ACN}]=1+K_{q} \tau_{0}[\mathrm{ACN}]
$$

In Eq. [1], $\mathrm{F}_{0}$ and $\mathrm{F}$ are the fluorescence emissions without and with quencher, respectively; $K_{S V}$ is the Stern-Volmer constant; $K_{q}$ is the bimolecular quenching constant; and $\tau_{0}$ is the lifetime of the fluorophore, which has been reported to be $3 \mathrm{~ns}$ for tryptophan (Lakowicz and Weber, 1973). Results shown in Table 4 confirmed that $K_{S V}$ can be calculated using a linear regression from the plot of $\mathrm{F}_{0} / \mathrm{F}$ against $\mathrm{ACN}$ concentration. The high coefficient of determination in Table $4\left(\mathrm{R}^{2}>\right.$ 0.98 ) indicates that quenching behavior was linear for all ACN, which is associated with a single fluorophore accessible to the quencher (Lakowicz, 2013). Moreover, the $K_{q}$ values of all ACN were larger than $10^{10} M^{-1} \mathrm{~s}^{-1}$, which is the value for diffusion-limited quenching in water (Lakowicz, 2013), strongly suggesting that the WPI-ACN interactions were of the binding-related type. This hypothesis was reinforced by the fact that $K_{q}$ showed no increase with higher temperatures. These results are consistent with published literature reporting static quenching of WP by ACN (Chung et al., 2015; He et al., 2016b; Khalifa et al., 2018).

As the quenching was likely a result of binding interactions, the apparent binding constants $\left(K_{a}\right)$ at different temperatures were calculated using the double logarithmic Stern-Volmer equation, Eq. [2] (Chung et al., 2015; He et al., 2016a; Stănciuc et al., 2017):

$$
\log \frac{\mathrm{F}_{0}-\mathrm{F}}{\mathrm{F}}=\log K_{a}+\mathrm{nLog}[\mathrm{ACN}]
$$

Results presented in Table 4 showed that analyses using Eq. [2] as a regression model had good linearity, denoted by high coefficients of determination $\left(\mathrm{R}^{2}\right.$ $>0.90)$ even at higher temperatures. The $K_{a}$ values observed for ACN were lower than those reported for complexation of $\beta$-LG with hydroxycinnamic acids

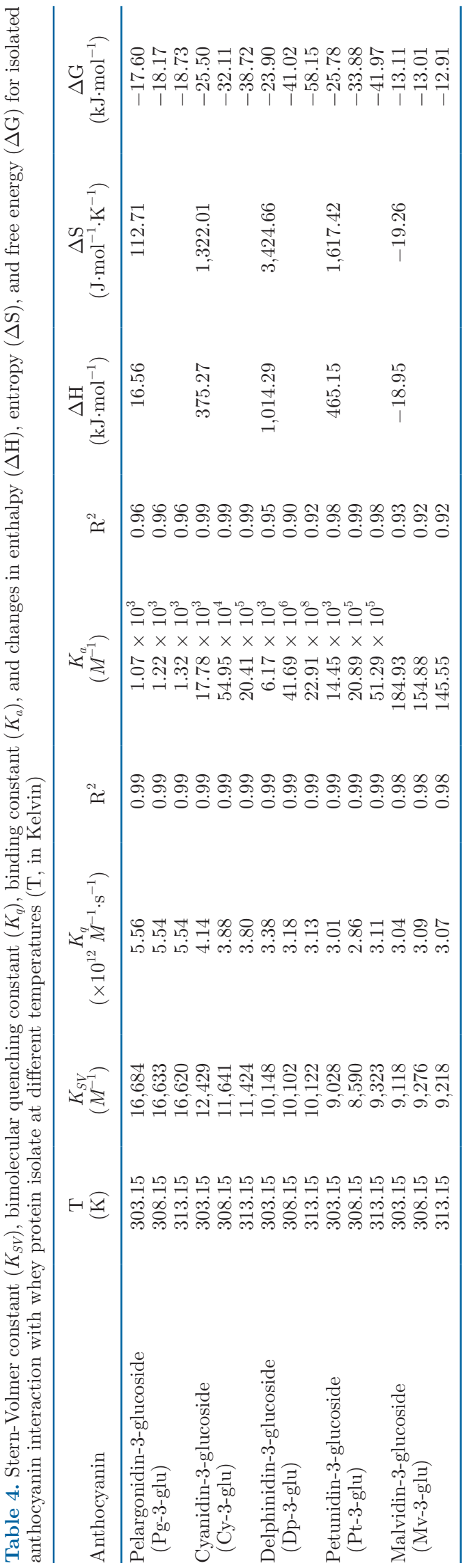


(Zhang et al., 2014) and hydroxybenzoic acids (Wu et al., 2018). However, they were similar to those reported for gallic acid and epigallocatechin gallate with WP (Cao and Xiong, 2017). Moreover, the $K_{a}$ values in this study were higher than those previously reported for BC ACN with WP (Chung et al., 2015), possibly because this study used isolated ACN rather than crude extracts. Results showed that $K_{a}$ increased with temperature for all ACN except mv-3-glu, suggesting that for most ACN, higher temperature promoted more stable complexation. This higher $K_{a}$ may be explained by a temperature-induced unfolding of globular proteins, such as $\beta$-LG (Stănciuc et al., 2012), resulting in stronger interactions with ACN. This is consistent with previous reports on the interaction of $\mathrm{WP}$ and $\mathrm{ACN}$ (Chung et al., 2015; Stănciuc et al., 2017) and may also explain the stronger protective effect of preheated WP over native WP previously reported (Chung et al., 2015; He et al., 2016a). The behavior of mv-3-glu was different than behaviors observed for pg-3-glu, cy3-glu, dp-3-glu, and pt-3-glu under the experimental conditions of this study; however, it was similar to the behavior reported for mv-3-glu with caseins (He et al., 2016b). The results of the present study suggest that the chemical structure of the ACN plays an important role in its complexation with WP. This is supported by a similar study showing that the chemical structures of phenolic compounds have significant influence on their affinity with proteins (Wu et al., 2018).

The effect of hydroxylations on the $\mathrm{B}$ ring of $\mathrm{ACN}$ was evaluated using pg-3-glu (1 OH group on position $\left.4^{\prime}\right)$, cy-3-glu ( $2 \mathrm{OH}$ groups on positions $3^{\prime}$ and $4^{\prime}$ ), and dp3 -glu (3 OH groups on positions $3^{\prime}, 4^{\prime}$ and $\left.5^{\prime}\right)$. Results showed that pg-3-glu had a lower affinity for WPI than cy-3-glu, and dp-3-glu had a lower $K_{a}$ than cy-3-glu but a higher $K_{a}$ than pg-3-glu. This affinity for WPI based on the number of hydroxylations and their position in the molecule is remarkably consistent with previous literature showing the same behavior for hydroxylated phenolic compounds and $\beta$-LG (Wu et al., 2018). Moreover, the effect of methoxylations in the ACN structure was also studied using dp-3-glu (no $\mathrm{OCH}_{3}$ groups in its structure), pt-3-glu (2 OH groups on positions $3^{\prime}$ and $4^{\prime}$, and $1 \mathrm{OCH}_{3}$ group on position $\left.5^{\prime}\right)$, and mv-3-glu (1 OH group on position $4^{\prime}$ and $2 \mathrm{OCH}_{3}$ groups on positions $3^{\prime}$ and $\left.5^{\prime}\right)$. Results showed that when comparing dp-3-glu and pt-3-glu, methoxylation on position $5^{\prime}$ resulted in a higher affinity for WP. When comparing pt-3-glu and mv-3-glu, the substitution of the hydroxyl group on position $3^{\prime}$ for a methoxy group resulted in a lower affinity for WP. This behavior, again, is consistent with that reported for phenolic compounds with similar substitu- tion patterns and $\beta-\mathrm{LG}$ (Wu et al., 2018). However, these results are contradictory to those reported for phenolics and human serum albumin and ovalbumin (Cao et al., 2013). These differences may be attributed to structural differences in the phenolic compounds and the proteins used in this study.

\section{Thermodynamic Parameters for the Interaction Between WP and ACN}

To further understand the type of binding interactions between WP and ACN, thermodynamic parameters were calculated using the Van't Hoff equation, Eq. [3]:

$$
\ln K_{a}=-\frac{\Delta \mathrm{H}}{\mathrm{RT}}+\frac{\Delta \mathrm{S}}{\mathrm{R}},
$$

where $K_{a}$ is the apparent binding constant calculated using Eq. [2], $\Delta \mathrm{H}$ is the enthalpy change, $\Delta \mathrm{S}$ is the entropy change, $\mathrm{R}$ is the gas constant, and $\mathrm{T}$ is the absolute temperature in Kelvin. The change in free energy $(\Delta G)$ of each WP-ACN system was calculated using Eq. [4] and the $\Delta \mathrm{H}$ and $\Delta \mathrm{S}$ values obtained from Eq. [3]:

$$
\Delta \mathrm{G}=\Delta \mathrm{H}-\mathrm{T} \Delta \mathrm{S} .
$$

Results presented in Table 3 showed that both $\Delta \mathrm{H}$ and $\Delta \mathrm{S}$ increased with increasing number of hydroxylations and decreased with increasing number of methoxylations. These patterns again confirm that complexation of ACN with WP is influenced by the chemical structure of the pigment. The $\Delta \mathrm{H}$ values showed that complex formation was an endothermic process, except for mv-3-glu, which was exothermic. These differences seem to indicate that the type of bond formed between the protein and the ACN may differ depending on the pigment structure. Ross and Subramanian (1981) classified different types of bonding based on $\Delta H$ and $\Delta S$ values. These results showed that for pg-3-glu, cy-3glu, dp-3-glu, and pt-3-glu, interactions with WP are predominantly dominated by hydrophobic interactions $(\Delta \mathrm{H}>0, \Delta \mathrm{S}>0)$; for mv-3-glu, interactions with WP are dominated by Van der Waals forces or hydrogen bonding $(\Delta \mathrm{H}<0, \Delta \mathrm{S}<0)$. Moreover, $\Delta \mathrm{G}$ values were negative for all WP-ACN complexes, indicating that complex formation was a spontaneous process. This is similar to other published literature on WP-ACN complex formation (Chung et al., 2015; Khalifa et al., 2018). 


\section{In Silico Modeling of WP-ACN Interactions}

In silico molecular docking simulations were used as a complementary tool to visible and fluorescence spectroscopy, allowing for the visualization of potential differences in the $\beta$-LG-ACN interaction. As shown in Figure 3, ACN positioning gives some light on why acylation did not play a significant role in the WP-ACN interaction. Modeling showed that the acyl moiety is positioned toward the outer part of the hydrophobic pocket of the protein, far from any amino acid residue. This could explain why the removal of the acyl group did not result in significant spectral changes in model solutions colored with ACN and copigmented with WP.
Although modeling for pg-3-glu and mv-3-glu showed that they docked in a similar position, analysis showed that the amino acid ends with which they interacted were different. Based on their electronic properties, amino acids can be classified into 5 categories: strong electron donors $(\mathrm{O})$, weak electron donors $(\mathrm{U})$, ambivalent (Z), weak electron acceptor (B), and strong electron acceptor (X; Dwyer, 2001). Proximity analysis in Figure 3 showed that hydroxylations in the $\mathrm{B}$ ring of pg-3-glu and dp-3-glu potentially interacted with a glutamic acid residue (Glu62) and an aspartic acid residue (Asp85), respectively. Both Glu62 and Asp85 are strong electron donors $(\mathrm{O})$. On the other side, methoxylations in the $\mathrm{B}$ ring of pt-3-glu and mv-3-glu interacted with

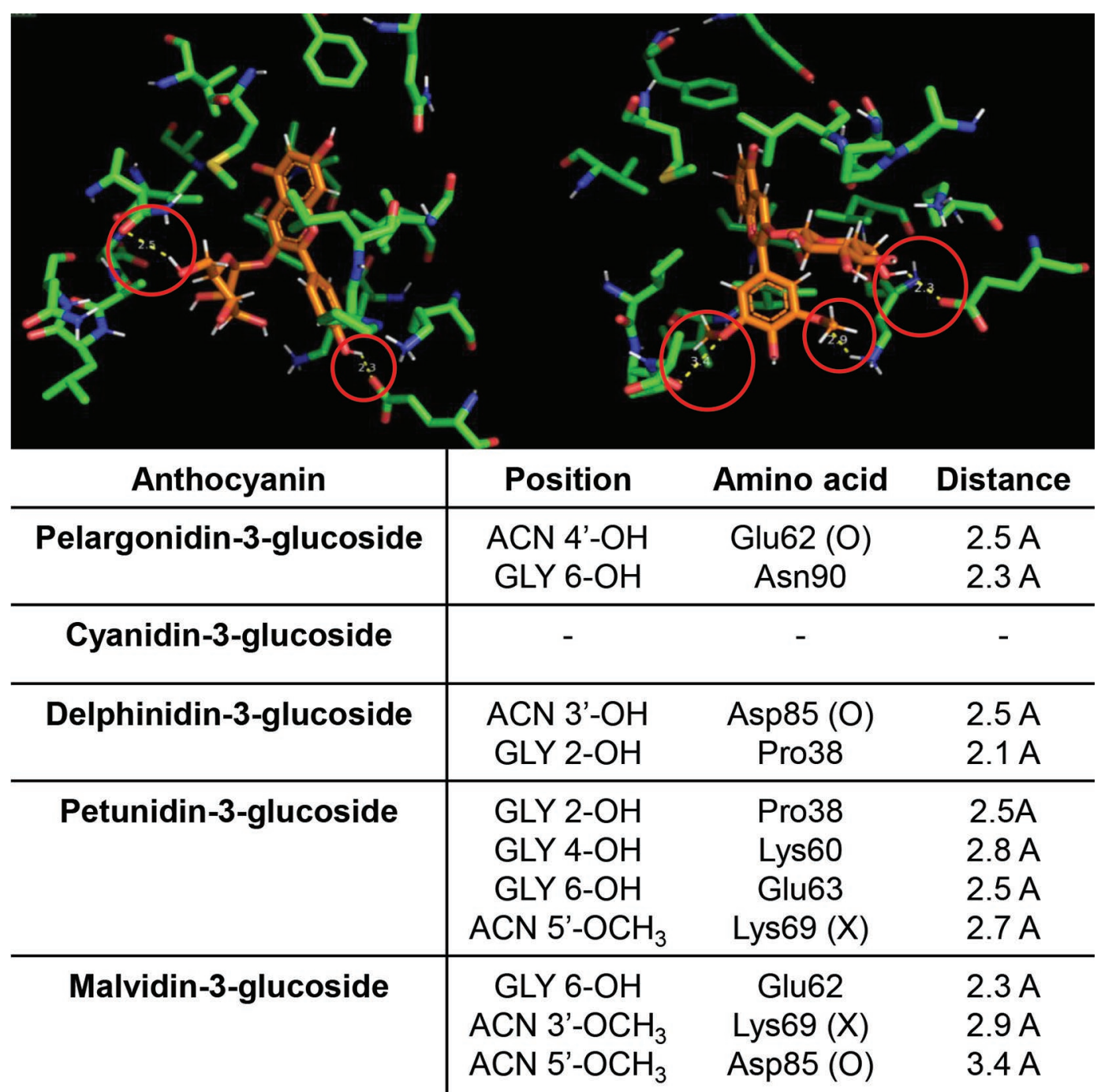

Figure 3. Representative in silico molecular docking of $\beta$-LG with pelargonidin-3-glucoside (left-hand column) and malvidin-3-glucoside (right-hand column). Potential interaction sites of anthocyanin-3-glucosides with AA residues are denoted by red circles, and respective distances were measured using PyMOL Molecular Graphics System (Schrödinger LLC). ACN = aglycon moiety, GLY = glycosylation moiety, -OH = hydroxylation, $-\mathrm{OCH}_{3}=$ methoxylation. $\mathrm{Glu}=$ glutamic acid, Asp = aspartic acid. $\mathrm{O}=$ strong electron donor, $\mathrm{X}=$ strong electron acceptor. 
lysine residues (Lys69), which are strong electron acceptors (X). These differences in the electronic properties of amino acids participating in the complexation process between WP and ACN may be the cause of the changes in the flavylium cation-chalcone equilibrium, thus explaining the dissimilar hypochromic and hyperchromic changes observed.

\section{CONCLUSIONS}

Addition of WPI to model solutions colored with ACN resulted in concentration-dependent hyperchromic effects and noticeable improvements in the tinctorial strength of ACN. However, not all ACN interacted in the same manner with WP-with methoxylated ACN showing larger absorption increments than nonmethoxylated ones due to a different type of interaction. The complexation process was spontaneous and predominantly dominated by hydrophobic interactions for most ACN 3-glucosides, except for mv-3-glu that interacted with WP via Van der Waals forces or hydrogen bonding. Modeling results showed that the electronic nature of the AA ends involved in the WP-ACN interaction may explain the different types of interactions observed. This study demonstrated that WP can be used as a color improving food additive when used in small concentrations. The WP effectiveness will depend on the ACN structural characteristics, highlighting the importance of ACN source selection for specific applications such as in the dairy industry.

\section{ACKNOWLEDGMENTS}

This research was supported in part by FONDECYT-CONCYTEC (Lima, Peru, grant number 225-2015-FONDECYT) and the USDA National Institute of Food and Agriculture (Washington, DC, Hatch Project OHO01423, Accession Number 1014136). The authors have not stated any conflicts of interest.

\section{REFERENCES}

Albani, J. R., J. Vogelaer, L. Bretesche, and D. Kmiecik. 2014. Tryptophan 19 residue is the origin of bovine $\beta$-lactoglobulin fluorescence. J. Pharm. Biomed. Anal. 91:144-150. https://doi.org/10 $.1016 /$ j.jpba.2013.12.015.

Ali, M., T. Homann, M. Khalil, H. P. Kruse, and H. Rawel. 2013. Milk whey protein modification by coffee-specific phenolics: Effect on structural and functional properties. J. Agric. Food Chem. 61:6911-6920. https://doi.org/10.1021/jf402221m.

Bateman, B., J. O. Warner, E. Hutchinson, T. Dean, P. Rowlandson, C. Gant, J. Grundy, C. Fitzgerald, and J. Stevenson. 2004. The effects of a double blind, placebo controlled, artificial food colourings and benzoate preservative challenge on hyperactivity in a general population sample of preschool children. Arch. Dis. Child. 89:506-511. https://doi.org/10.1136/adc.2003.031435.
Burrows, A. 2009. Palette of our palates: A brief history of food coloring and its regulation. Compr. Rev. Food Sci. Food Saf. 8:394-408. https://doi.org/10.1111/j.1541-4337.2009.00089.x.

Cao, H., X. Jing, D. Wu, and Y. Shi. 2013. Methylation of genistein and kaempferol improves their affinities for proteins. Int. J. Food Sci. Nutr. 64:437-443. https://doi.org/10.3109/09637486.2012 .759186 .

Cao, Y., and Y. L. Xiong. 2017. Interaction of whey proteins with phenolic derivatives under neutral and acidic $\mathrm{pH}$ conditions. J. Food Sci. 82:409-419. https://doi.org/10.1111/1750-3841.13607.

Chatham, L. A., J. E. Howard, and J. A. Juvik. 2020. A natural colorant system from corn: Flavone-anthocyanin copigmentation for altered hues and improved shelf life. Food Chem. 310:125734. https://doi.org/10.1016/j.foodchem.2019.125734.

Chen, L. J., and G. Hrazdina. 1982. Structural transformation reactions of anthocyanins. Experientia 38:1030-1032. https://doi.org/ 10.1007/BF01955348.

Chung, C., T. Rojanasasithara, W. Mutilangi, and D. J. McClements. 2015. Enhanced stability of anthocyanin-based color in model beverage systems through whey protein isolate complexation. Food Res. Int. 76:761-768. https://doi.org/10.1016/j.foodres.2015.07 .003 .

Crisosto, C. H., G. M. Crisosto, and P. Metheney. 2003. Consumer acceptance of "Brooks" and "Bing" cherries is mainly dependent on fruit SSC and visual skin color. Postharvest Biol. Technol. 28:159167. https://doi.org/10.1016/S0925-5214(02)00173-4.

Dangles, O., C. Stoeckel, M. C. Wigand, and R. Brouillard. 1992a. Two very distinct types of anthocyanin complexation: Copigmentation and inclusion. Tetrahedron Lett. 33:5227-5230. https://doi .org/10.1016/S0040-4039(00)79139-8.

Dangles, O., M. C. Wigand, and R. Brouillard. 1992b. Anthocyanin anti-copigment effect. Phytochemistry 31:3811-3812. https://doi .org/10.1016/S0031-9422(00)97533-2.

Dwyer, D. S. 2001. Electronic properties of the amino acid side chains contribute to the structural preferences in protein folding. J. Biomol. Struct. Dyn. 18:881-892. https://doi.org/10.1080/07391102 .2001.10506715.

Eiro, M. J., and M. Heinonen. 2002. Anthocyanin color behavior and stability during storage: Effect of intermolecular copigmentation. J. Agric. Food Chem. 50:7461-7466. https://doi.org/10.1021/ jf0258306.

Farr, J. E., and M. M. Giusti. 2017. ColorBySpectra for academic use. Accessed Aug. 5, 2020. https://techlicenseexpress.com/products/ colorbyspectra.

Farr, J. E., G. T. Sigurdson, and M. M. Giusti. 2018. Influence of cyanidin glycosylation patterns on carboxypyranoanthocyanin formation. Food Chem. 259:261-269. https://doi.org/10.1016/j .foodchem.2018.03.117.

Giusti, M. M., and R. E. Wrolstad. 1996. Characterization of red radish anthocyanins. J. Food Sci. 61:322-326. https://doi.org/10 $.1111 /$ j.1365-2621.1996.tb14186.x.

Giusti, M. M., and R. E. Wrolstad. 2001. Characterization and measurement of anthocyanins by UV-visible spectroscopy. Curr. Protoc. Food Anal. Chem. 1:F1.2.1-F2.13. https://doi.org/10.1002/ 0471142913.faf0102s00.

Giusti, M. M., and R. E. Wrolstad. 2003. Acylated anthocyanins from edible sources and their applications in food systems. Biochem. Eng. J. 14:217-225. https://doi.org/10.1016/S1369-703X(02)00221 -8 .

He, J., and M. M. Giusti. 2010. Anthocyanins: Natural colorants with health-promoting properties. Annu. Rev. Food Sci. Technol. 1:163187. https://doi.org/10.1146/annurev.food.080708.100754.

He, Z., M. Xu, M. Zeng, F. Qin, and J. Chen. 2016a. Preheated milk proteins improve the stability of grape skin anthocyanins extracts. Food Chem. 210:221-227. https://doi.org/10.1016/j.foodchem .2016.04.116.

He, Z., M. Xu, M. Zeng, F. Qin, and J. Chen. 2016b. Interactions of milk $\alpha$ - and $\beta$-casein with malvidin-3-O-glucoside and their effects on the stability of grape skin anthocyanin extracts. Food Chem. 199:314-322. https://doi.org/10.1016/j.foodchem.2015.12.035. 
Hsiao, Y. P., H. L. Huang, W. W. Lai, J. G. Chung, and J. H. Yang. 2009. Antiproliferative effects of lactic acid via the induction of apoptosis and cell cycle arrest in a human keratinocyte cell line (HaCaT). J. Dermatol. Sci. 54:175-184. https://doi.org/10.1016/j .jdermsci.2009.02.012.

Jia, Z., M. Zheng, F. Tao, W. Chen, G. Huang, and J. Jiang. 2016. Effect of covalent modification by (-)-epigallocatechin-3-gallate on physicochemical and functional properties of whey protein isolate. Lebensm. Wiss. Technol. 66:305-310. https://doi.org/10.1016/j .lwt.2015.10.054.

Jing, P., and M. M. Giusti. 2005. Characterization of anthocyanin-rich waste from purple corncobs (Zea mays L.) and its application to color milk. J. Agric. Food Chem. 53:8775-8781. https://doi.org/ 10.1021/jf051247o.

Kanha, N., S. Surawang, P. Pitchakarn, J. M. Regenstein, and T. Laokuldilok. 2019. Copigmentation of cyanidin 3-O-glucoside with phenolics: Thermodynamic data and thermal stability. Food Biosci. 30:100419. https://doi.org/10.1016/j.fbio.2019.100419.

Khalifa, I., R. Nie, Z. Ge, K. Li, and C. Li. 2018. Understanding the shielding effects of whey protein on mulberry anthocyanins: Insights from multispectral and molecular modelling investigations. Int. J. Biol. Macromol. 119:116-124. https://doi.org/10.1016/j .ijbiomac.2018.07.117.

Lakowicz, J. R. 2013. Principles of Fluorescence Spectroscopy. Springer Science and Business Media.

Lakowicz, J. R., and G. Weber. 1973. Quenching of protein fluorescence by oxygen. Detection of structural fluctuations in proteins on the nanosecond time scale. Biochemistry 12:4171-4179. https:/ /doi.org/10.1021/bi00745a021.

Liang, L., H. A. Tajmir-Riahi, and M. Subirade. 2008. Interaction of $\beta$-lactoglobulin with resveratrol and its biological implications. Biomacromolecules 9:50-56. https://doi.org/10.1021/bm700728k.

Mazza, G., and R. Brouillard. 1990. The mechanism of co-pigmentation of anthocyanins in aqueous solutions. Phytochemistry 29:1097-1102. https://doi.org/10.1016/0031-9422(90)85411-8.

McCann, D., A. Barrett, A. Cooper, D. Crumpler, L. Dalen, K. Grimshaw, E. Kitchin, K. Lok, L. Porteous, E. Prince, E. Sonuga-Barke, J. O. Warner, and J. Stevenson. 2007. Food additives and hyperactive behaviour in 3-year-old and 8/9-year-old children in the community: A randomised, double-blinded, placebo-controlled trial. Lancet 370:1560-1567. https://doi.org/10.1016/S0140 -6736(07)61306-3.

Mokrzycki, W., and M. Tatol. 2011. Color difference Delta E-A survey. Mach. Graph. Vis. 20:383-411.

Obón, J. M., M. R. Castellar, M. Alacid, and J. A. Fernández-López. 2009. Production of a red-purple food colorant from Opuntia stric$t a$ fruits by spray drying and its application in food model systems. J. Food Eng. 90:471-479. https://doi.org/10.1016/j.jfoodeng.2008 .07 .013

Ozdal, T., E. Capanoglu, and F. Altay. 2013. A review on protein-phenolic interactions and associated changes. Food Res. Int. 51:954970. https://doi.org/10.1016/j.foodres.2013.02.009.

Rawel, H. M., J. Kroll, and U. C. Hohl. 2001. Model studies on reactions of plant phenols with whey proteins. Nahrung 45:72-81. https:// doi.org/10.1002/1521-3803(20010401)45:2<72::AIDFOOD72>3.0.CO;2-U.

Rodríguez, S. D., M. von Staszewski, and A. M. R. Pilosof. 2015. Green tea polyphenols-whey proteins nanoparticles: Bulk, interfacial and foaming behavior. Food Hydrocoll. 50:108-115. https:// doi.org/10.1016/j.foodhyd.2015.04.015.

Rodriguez-Saona, L. E., and R. E. Wrolstad. 2001. Extraction, isolation, and purification of anthocyanins. Unit F1.1. Handbook of Food Analytical Chemistry. R. E. Wrolstad and S. J. Schwartz, ed. John Wiley and Sons Ltd.

Rohn, S., H. M. Rawel, and J. Kroll. 2004. Antioxidant activity of protein-bound quercetin. J. Agric. Food Chem. 52:4725-4729. https:/ /doi.org/10.1021/jf0496797.
Ross, P. D., and S. Subramanian. 1981. Thermodynamics of protein association reactions: Forces contributing to stability. Biochemistry 20:3096-3102. https://doi.org/10.1021/bi00514a017.

Santos, H., D. L. Turner, J. C. Lima, P. Figueiredo, F. S. Pina, and A. L. Maçanita. 1993. Elucidation of the multiple equilibria of malvin in aqueous solution by one- and two-dimensional NMR. Phytochemistry 33:1227-1232. https://doi.org/10.1016/0031 -9422(93)85054-U.

Serafini, M., M. F. Testa, D. Villaño, M. Pecorari, K. van Wieren, E. Azzini, A. Brambilla, and G. Maiani. 2009. Antioxidant activity of blueberry fruit is impaired by association with milk. Free Radic. Biol. Med. 46:769-774. https://doi.org/10.1016/j.freeradbiomed .2008.11.023.

Sigurdson, G. T., P. Tang, and M. M. Giusti. 2017. Natural colorants: Food colorants from natural sources. Annu. Rev. Food Sci. Technol. 8:261-280. https://doi.org/10.1146/annurev-food-030216 -025923 .

Stănciuc, N., I. Aprodu, G. Râpeanu, and G. Bahrim. 2012. Fluorescence spectroscopy and molecular modeling investigations on the thermally induced structural changes of bovine $\beta$-lactoglobulin. Innov. Food Sci. Emerg. Technol. 15:50-56. https://doi.org/10.1016/ j.ifset.2012.03.001.

Stănciuc, N., M. Turturică, A. M. Oancea, V. Barbu, E. Ioniţă, I. Aprodu, and G. Râpeanu. 2017. Microencapsulation of anthocyanins from grape skins by whey protein isolates and different polymers. Food Bioprocess Technol. 10:1715-1726. https://doi.org/10 $.1007 / \mathrm{s} 11947-017-1938-8$.

Wallace, T. C., and M. M. Giusti. 2008. Determination of color, pigment, and phenolic stability in yogurt systems colored with nonacylated anthocyanins from Berberis boliviana L. as compared to other natural/synthetic colorants. J. Food Sci. 73:C241-C248. https://doi.org/10.1111/j.1750-3841.2008.00706.x.

Wilska-Jeszka, J., and A. Korzuchowska. 1996. Anthocyanins and chlorogenic acid copigmentation-Influence on the colour of strawberry and chokeberry juices. Eur. Food Res. Technol. 203:38-42. https://doi.org/10.1007/BF01267767.

Wrolstad, R. E. 2004. Anthocyanin pigments-Bioactivity and coloring properties. J. Food Sci. 69. https://doi.org/10.1111/j.1365 -2621.2004.tb10709.x.

Wu, S., Y. Zhang, F. Ren, Y. Qin, J. Liu, J. Liu, Q. Wang, and H. Zhang. 2018. Structure-affinity relationship of the interaction between phenolic acids and their derivatives and $\beta$-lactoglobulin and effect on antioxidant activity. Food Chem. 245:613-619. https:// doi.org/10.1016/j.foodchem.2017.10.122.

Yamada, T., T. Komiya, and M. Akaki. 1980. Formation of an inclusion complex of anthocyanin with cyclodextrin. Agric. Biol. Chem. 44:1411-1413. https://doi.org/10.1080/00021369.1980.10864141.

Zhang, H., D. Yu, J. Sun, H. Guo, Q. Ding, R. Liu, and F. Ren. 2014. Interaction of milk whey protein with common phenolic acids. J. Mol. Struct. 1058:228-233. https://doi.org/10.1016/j.molstruc .2013.11.009.

Zhao, X., B. W. Ding, J. W. Qin, F. He, and C. Q. Duan. 2020. Intermolecular copigmentation between five common 3-O-monoglucosidic anthocyanins and three phenolics in red wine model solutions: The influence of substituent pattern of anthocyanin B ring. Food Chem. 326:126960. https://doi.org/10.1016/j.foodchem .2020 .126960

\section{ORCIDS}

G. Miyagusuku-Cruzado $\odot$ https://orcid.org/0000-0002-4420-6022

R. Jiménez-Flores @ https://orcid.org/0000-0003-4905-5021

M. M. Giusti @ https://orcid.org/0000-0002-2348-3530 